\title{
Changes in salivary biomarkers of oxidative status in calves at weaning and grouping
}

\author{
Camila Peres Rubio ${ }^{1 *}$, Damián Escribano ${ }^{1}$, Eva Mainau ${ }^{1}$, José Joaquin Cerón ${ }^{2}$, Elena Navarro ${ }^{1}$ and \\ Xavier Manteca ${ }^{1}$
}

\begin{abstract}
Background: Saliva is being increasingly used as a sample for measuring biomarkers in several species and shows a high potential of use to detect and monitor stress. The weaning and grouping in dairy calves are a particularly stressful time. Therefore, the objectives of this study were to evaluate a panel of antioxidant and oxidant biomarkers in the saliva of calves on the day of weaning (W0), 2 days after weaning or milk withdrawal ( $W+2)$, and 4 days after grouping $(G+4)$. In addition, to verify if cortisol and oxytocin concentrations are related to the biomarkers measured.

Results: Salivary cupric reducing antioxidant capacity (CUPRAC), ferric reducing ability of saliva (FRAS), Trolox equivalent antioxidant capacity (TEAC), advanced oxidation protein products (AOPP), and ferrous oxidation-xylenol orange (FOX) were significantly higher $(P<0.02) 4$ days after grouping than the day of weaning and 2 days after. The increases were 50 and 54\% for CUPRAC, 93 and 116\% for FRAS, 117 and 135\% for TEAC, 22 and 49\% for AOPP and 10 and 5\% for FOX in comparison with weaning and 2 days after, respectively. In addition, oxytocin and cortisol showed significant negative and positive correlations $(P<0.05)$ respectively with the biomarkers of oxidative status.
\end{abstract}

Conclusions: Our results showed that calves after grouping show increases in antioxidants and oxidants concentrations, indicating that a balance between these molecules has been tried to maintain during this stressful situation. The dynamic changes of biomarkers of oxidative status should be explored and characterised in other stressful conditions.

Keywords: Antioxidants, Cupric, Ferric, Oxidant, Stress

\section{Background}

Biomarkers of oxidative status include those analytes that can measure or estimate antioxidant or oxidant compounds. These biomarkers can detect situations in which the antioxidant system cannot counteract the overproduction of oxidants, such as reactive oxygen species (ROS) [1]. These situations can lead to oxidative damage of molecules such as lipids, proteins, DNA and RNA, and are related to various diseases in humans and animals [2-4].

\footnotetext{
*Correspondence: camila.peres@uab.cat

1 Department of Animal and Food Science, School of Veterinary Science,

Universitat Autònoma de Barcelona, 08193 Cerdanyola del Vallès,

Barcelona, Spain

Full list of author information is available at the end of the article
}

Traditionally oxidative stress biomarkers in ruminants have been measured in blood, and their variations have been associated with diseases. For example, changes in these biomarkers have been described in cows with metabolic disorders such as ketosis [5], or reproductive disorders like ovarian follicular cyst and endometritis $[6$, 7], in bulls with severe symptoms of foot-and-mouth disease [8], and in sheep during parasitism infection $[9,10]$. In addition, different situations that can produce stress in ruminants have also been associated with changes in antioxidant and oxidant biomarkers, a condition also called "oxidative eustress" [11]. For example, in lactating dairy cows during thermal stress [12] or the transition from late lactation and into the early dry period [13] and shearing in sheep [14]. The mechanism involved remains unclear, but it seems the changes in oxidative biomarkers 
could be, in part, mediated by stress-related hormones such as cortisol $[15,16]$.

Saliva is being increasingly used as a sample to measure biomarkers in several species [17-19]. This is due to various facts, such as its non-invasive and inexpensive collection and composition, that can reflect dynamic changes in the body [20]. In particular, biomarkers of oxidative status such as total antioxidant capacity (Trolox equivalent antioxidant capacity [TEAC], ferric reducing ability of saliva [FRAS], cupric reducing antioxidant capacity [CUPRAC]), uric acid, thiol, advanced oxidation protein products (AOPP) and hydrogen peroxide $\left(\mathrm{H}_{2} \mathrm{O}_{2}\right)$ have been measured in the saliva of cows [21, 22].

The weaning in dairy calves is a period of diet change and a new physical and social environment [23] which can result in adverse effects on both production and wellbeing [24], being a particularly stressful time [25]. In the same way, the grouping of calves can lead to aggression and social stress $[26,27]$.

In this study, we hypothesised that the biomarkers of oxidative stress could change in the saliva of calves during stress situations such as weaning and grouping, and that these changes could be related to those produced in biomarkers of welfare and stress such as oxytocin and cortisol. Therefore, the objectives of this work were to evaluate a panel of antioxidant (CUPRAC, FRAS, TEAC, and uric acid) and oxidant biomarkers (AOPP, ferrous oxidation-xylenol orange [FOX] and peroxide-activity [POX-Act]) in the saliva of calves on the day of the weaning (W0), 2 days after weaning $(\mathrm{W}+2)$ or milk withdrawal and 4 days after grouping $(G+4)$. In addition, the correlation of these biomarkers with stress biomarkers such as cortisol and oxytocin were evaluated.

\section{Results}

\section{Biomarkers of oxidative status}

The results obtained in the measured biomarkers of oxidative status in the calves throughout the study are presented in Fig. 1. On the antioxidant side, a significant increase $(P<0.021)$ was observed 4 days after grouping calves $(\mathrm{G}+4)$ when compared to the day of weaning (W0) and 2 days after weaning or milk withdrawal $(\mathrm{W}+2)$ in the concentrations of CUPRAC (which showed a 50 and $54 \%$ increase compared with W0 and $\mathrm{W}+2$, respectively), FRAS (93 and $116 \%$ increase compared with $\mathrm{WO}$ and $\mathrm{W}+2$, respectively), and TEAC (117 and 135\% increase compared with W0 and W +2 , respectively). Uric acid did not show differences between the time-points of the study $(P>0.05)$. Regarding the oxidants, the POX-Act results were below the detection limit in saliva and data is not shown. Salivary AOPP concentrations were significantly higher at $\mathrm{G}+4$ than $\mathrm{W}+2(49 \%$ increase; $P=0.002)$. FOX showed higher concentrations at G+4 when compared with W0 $(10 \%$ increase; $P=0.021$ ).

\section{Cortisol}

The results of salivary cortisol are shown in Fig. 2. No significant changes were observed in the cortisol concentrations during all the periods of study $(P<0.05)$.

\section{Correlation study}

The correlations between the different biomarkers studied, the salivary cortisol and the previously reported values of salivary oxytocin [16] are shown in Table 1. Significant and negative correlations with oxytocin were found with CUPRAC $(r=-0.43, \quad P<0.0001)$, FRAS $(r=-0.38, P<0.0006)$, TEAC $(r=-0.40, P<0.0003)$ and AOPP $(r=-0.30, P=0.007)$. Regarding cortisol, positive and significant correlations were observed with CUPRAC $(r=0.25, P=0.028)$, FRAS $(r=0.29, P=0.011)$, TEAC $(r=0.31, P=0.005)$ and AOPP $(r=0.40, P=0.0003)$.

\section{Discussion}

This study is the first that determines a panel of biomarkers of oxidative status in the saliva of calves during productive and stressful situations such as weaning and grouping. Also, the possible relationship between those biomarkers and biomarkers of stress and welfare such as cortisol and oxytocin in the saliva of calves are evaluated.

The increase in the oxidant biomarkers observed in our work in the saliva of the calves 4 days after grouping would reflect an oxidative challenge. Possible links between stressful situations and the increase in oxidant compounds have been reported. It seems that excessive production of oxygen radicals through the higher formation of xanthine oxidase occurs during stressful stimuli [28]. In addition, any stressful situations can activate the hypothalamic-pituitary-adrenal axis followed by releasing the corresponding stress hormones such as cortisol and corticosterone [29], and increase the presence of ROS in cell cultures [30], which could be one of the mechanisms over stress led to the overproduction of oxidants as observed in this study in saliva. In this line, increases in oxidants after stressful conditions in animals have been described, such as increased serum and plasma lipid peroxides in horses and calves after transportation [31, 32].

The increased antioxidant biomarkers found in our study would aim to counteract the oxidants' increase after the stressful condition. This has been described in previous reports in which stressful situations have been related to lipid peroxidation and consequently increased antioxidant activity as an adaptation to the stimulus [33, 34]. In agreement with our results, salivary oxidant and antioxidant biomarkers were 

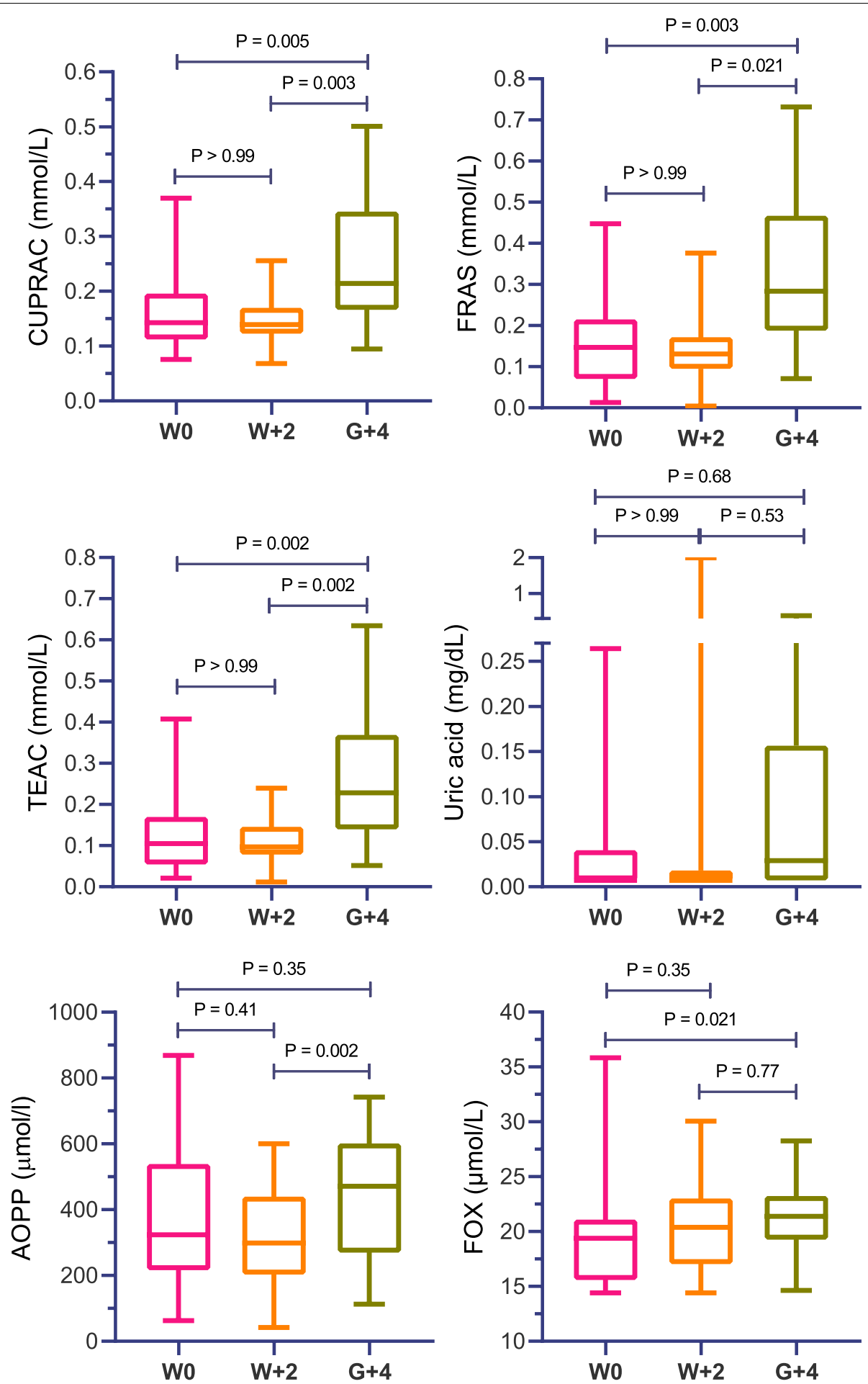

Fig. 1 Salivary cupric reducing antioxidant capacity (CUPRAC), ferric reducing ability of saliva (FRAS), Trolox equivalent antioxidant capacity (TEAC), uric acid, advanced oxidation protein products (AOPP), and ferrous oxidation-xylenol orange (FOX) of 25 calves on the day of weaning (W0), 2 days after weaning or milk withdrawal $(W+2)$ and 4 days after grouping calves $(G+4)$ 


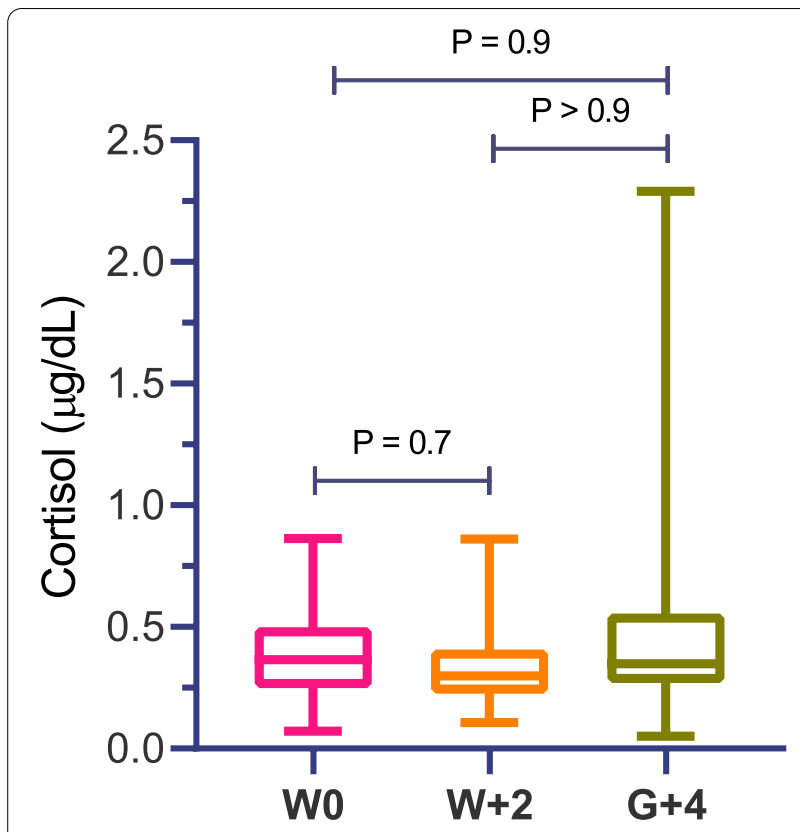

Fig. 2 Cortisol in saliva of calves on the day of the weaning (W0), 2 days after weaning or milk withdrawal $(W+2)$ and 4 days after grouping calves $(\mathrm{G}+4)$

Table 1 Correlation coefficients between oxidative status biomarkers and oxytocin and cortisol concentrations in the calves on the day of the weaning, after weaning and after grouping $(n=25)$

\begin{tabular}{lll}
\hline Biomarker of oxidative status & Oxytocin & Cortisol \\
\hline CUPRAC & $-0.435^{* * *}$ & $0.252^{*}$ \\
FRAS & $-0.385^{* * *}$ & $0.290^{*}$ \\
TEAC & $-0.402^{* * *}$ & $0.314^{* *}$ \\
Uric acid & -0.123 & -0.043 \\
AOPP & $-0.307^{* *}$ & $0.409^{* * *}$ \\
FOX & -0.189 & 0.014 \\
\hline
\end{tabular}

CUPRAC cupric reducing antioxidant capacity, FRAS ferric reducing ability of saliva, TEAC Trolox equivalent antioxidant capacity, AOPP advanced oxidation protein products, FOX ferrous oxidation-xylenol orange

${ }^{*} P<0.05$; ${ }^{*} P<0.01$; ${ }^{* *} P<0.001$

increased in sheep after an acute stress stimulus such as shearing [19]. In addition, increases in antioxidants in blood in stressful situations have been described in different species. For example, in dogs, the transportation triggered the activation of antioxidant response in serum [35]. In cows, increased oxidants and mobilisation of blood antioxidants have been reported after weaning and heat stress [36, 37]. The occurrence of a state of oxidative stress in calves may increase the potential and incidence of infectious and metabolic diseases, which denotes that the measurement of biomarkers of oxidative status would be of help to improve the management practices for these animals [36].

Oxytocin and cortisol hormones have been associated with positive and negative welfare, respectively, in calves and other species [16, 38-40]. In our study, although no significant change has been observed in the salivary cortisol, salivary oxytocin correlated negatively with the oxidant and antioxidant biomarkers, whereas cortisol showed a positive correlation. Although the correlations were moderate, they were all highly significant statistically. The positive correlation between the biomarkers of oxidative status and cortisol can be explained by the activation of the hypothalamic-pituitary-adrenal axis as described above, while the negative correlation with oxytocin could be due to its decrease in situations of stress or negative emotions [16].

This study has limitations that deserve consideration. The biomarkers results have not been corrected by salivary protein, and additional studies should be performed in the future about the possible need for correction by protein in biomarkers of oxidative status [41, 42]. Only females have been included in the experimental design, thence further investigation about the sex-specific differences in oxidative stress biomarkers in saliva should be done. Furthermore, the effects of the dietary changes during the experiment have not been considered, and ideally, a control group without grouping after weaning should have been included. Besides, studies including sampling times before than $24 \mathrm{~h}$ after weaning would allow evaluation of the oxidative response in an acute situation of weaning. In the same way, sampling in a longer time than 4 days of grouping would help to find when the concentrations of the biomarkers achieve the concentration observed at weaning.

\section{Conclusions}

In this report, we demonstrated a significant increase in oxidant biomarkers such as AOPP and FOX and antioxidant biomarkers such as CUPRAC, FRAS, and TEAC in the saliva of calves after a stressful situation like grouping. Based on these preliminary results, it could be concluded that stress situations would promote an increase in oxidant molecules, which is compensated by an increase in antioxidants, a situation that could be assessed by measuring biomarkers of oxidative status in the saliva. The dynamic changes of biomarkers of oxidative status should be explored and characterised in other stressful conditions. 


\section{Material and methods}

\section{Animals, housing, and general management}

The trial was in conformity with the ARRIVE guidelines [43]. Twenty-five Friesian female calves from multiparous and primiparous dams from a commercial farm were studied, split into 6 replicates (or weeks). Each replicate included from 2 to 7 calves. Calves were separated from dams as soon as possible after calving. The mean $\pm E S$ interval time between calving and calf-dam separation was $28.86 \pm 4.64 \mathrm{~min}$. Calves were moved from the maternity pen to individual hutches $(1.2 \mathrm{~m} \times 1.8 \mathrm{~m})$ that were bedded with straw within a 24-hutches, 6-row outdoor calf facility. As soon as calves were allocated in the hutches, they received $4 \mathrm{~L}$ of colostrum by oesophageal feeder tube. Then, calves fed pasteurized milk from the tank of the farm at 8:00 am and 4:00 pm by individual calf buckets. The following quantities were given to each calf: $6 \mathrm{~L} / \mathrm{d}$ from day 2 to day $14,8 \mathrm{~L} / \mathrm{d}$ from day 15 to day 42 , $6 \mathrm{~L} / \mathrm{d}$ from day 43 to day 49 and $4 \mathrm{~L} / \mathrm{d}$ from day 50 until weaning (gradual reduction of milk supply). In compliance with management practices on the farm, two criteria had to be met to wean calves: (1) calves with at least 8 weeks of life (from day 57 to day 63 after birth) and (2) calves with body weight $(\mathrm{BW}) \geq 80 \mathrm{Kg}$. Every Tuesday at $12: 00 \mathrm{pm}$, calves that were expected to be weaned (at least 8 weeks of life), were individually weighted. For calves meeting the criteria ( $\mathrm{BW} \geq 80 \mathrm{Kg}$ ), milk was withdrawn in the afternoon. Calves with $\mathrm{BW}<80 \mathrm{Kg}$, were fed milk (4L/day) until reached $80 \mathrm{Kg}$ for milk withdrawal. In the present work, 19 calves were weaned at 8 weeks of life and 6 calves needed one additional week for reaching at least $80 \mathrm{Kg}$ (9 weeks of life). Mean $\pm \mathrm{ES}$ of weight at weaning (milk withdrawal) was $87.01 \pm 0.78 \mathrm{Kg}$ and calves had $60.96 \pm 0.86$ days of life. After 3 days of milk withdrawal (Friday), calves were moved to a group pen of 8-10 calves each. Calves were transported within the same farm a distance of $1200 \mathrm{~m}$ by a small truck.

Freshwater was offered to calves starting at $1 \mathrm{~d}$ of age. Water was changed twice daily after milk administration. Calves had free access to a starter diet 1 week after birth. Calves were offered with a $300 \mathrm{~g}$ of starter diet 1 week after birth. Every day at 12:00 pm, the starter was checked and changed ensuring that calves were fed ad libitum. The starter diet was formulated based on NRC recommendations [44]. After grouping, calves were fed TMR mixed with the same starter diet for 15 days (See Additional file).

\section{Saliva collection}

Saliva samples were obtained from all animals at three time-points: on the day of the weaning (W0), 2 days after weaning or milk withdrawal $(W+2)$ and 7 days after weaning that corresponded to 4 days after grouping calves $(G+4)$. All samples were obtained between 7:00 and 8:00 am (before morning milk feeding or TMR mixed administration) (Fig. 3). Saliva collections were performed using a cotton swab attached through a Kocher clip, and calves were allowed to chew it for $1 \mathrm{~min}$. Then, the cotton swab was placed into Salivette tubes (Sarstedt, Aktienge-sellschaft \& Co. Nümbrecht, Germany) and centrifuged at $6000 \mathrm{rpm}$ for $12 \mathrm{~min}$. Approximately 1 to $2 \mathrm{~mL}$ of saliva from each cotton swab were stored in Eppendorf tubes and frozen at $-80^{\circ} \mathrm{C}$ until analysis.

\section{Oxidative stress biomarkers}

About antioxidant biomarkers determination, CUPRAC was measured as previously described [45] and is based on the capacity of the sample in reducing cupric to cuprous ion. The FRAS was performed following the method described by Benzie and Strain [46], which

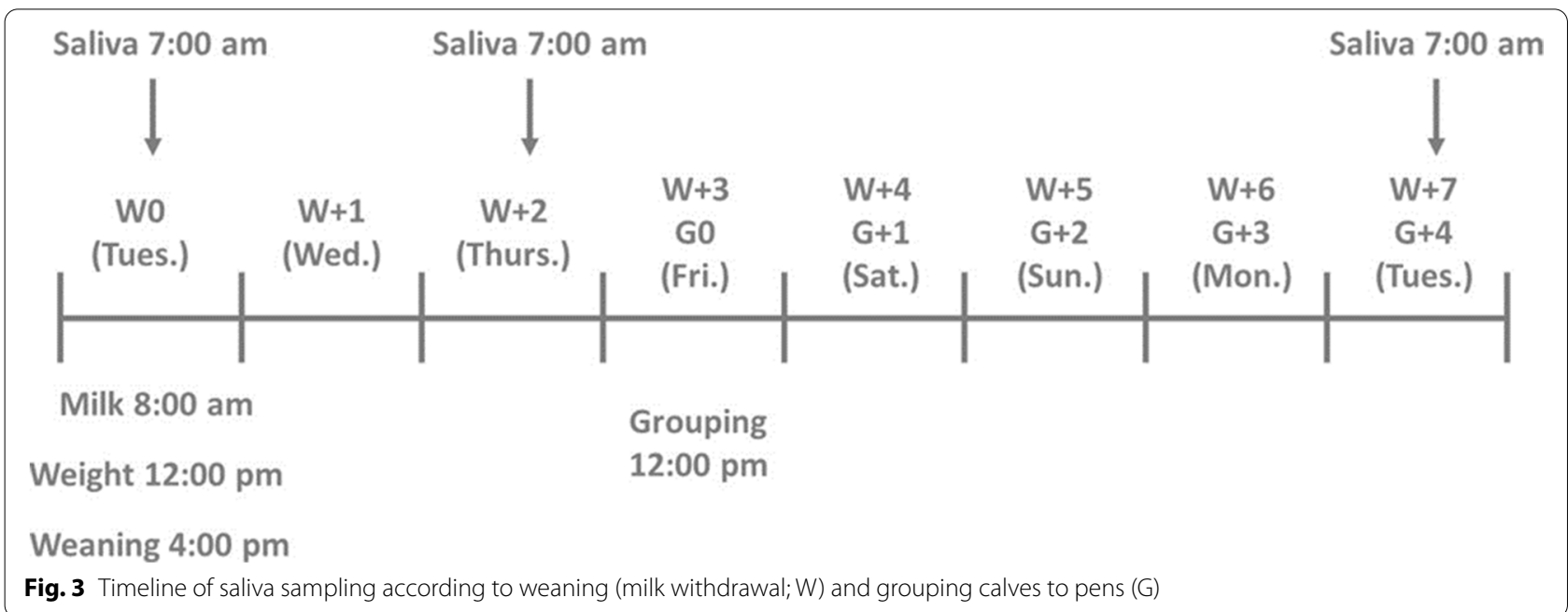


measures the ferric to ferrous ion reduction by the sample. The TEAC, based on the inhibition of the radical ABTS by the sample, was performed according to the assay described by Arnao et al. [47]. Finally, uric acid was measured using a commercially available spectrophotometric method (Uric Acid reagent OSR6698 Beckman Coulter AU analysers, Switzerland).

Regarding oxidant biomarkers evaluation, AOPP was based on the determination of oxidatively modified albumin and di-tyrosine containing cross-linked proteins as previously described [48]. The FOX was assessed based on the automatic determination of the ferrous oxidation by xylenol orange as described by Arab and Steghens [49]. Finally, the POX-Act was performed based on the oxidation of the chromogen substrate 3,5,3'5'-tetramethylbenzidine (TMB) by the reaction of horseradish peroxidase (HRP) with the sample as previously described [50].

All analyses were done at Murcia University using an automated biochemistry analyser (Olympus AU400 Automatic Chemistry Analyzer, Olympus Europe GmbH, Germany). All assays showed inter-and intra-assay imprecision of $<15 \%$ and showed high linearity $\left(r^{2}>0.95\right)$ after serial sample dilutions.

\section{Cortisol assay}

The cortisol concentration in saliva was determined by the use of a solid-phase, competitive chemiluminescent enzyme immunoassay that used a polyclonal rabbit anticortisol antibody (Immulite/Immulite 1000 cortisol; Siemens Medical Solutions Diagnostics, Deerfield, IL, USA).

\section{Statistical analysis}

Statistical analyses were performed using Graph Pad Software Inc. (GraphPad Prism, version 6 for Windows, Graph Pad Software Inc., San Diego, USA). Statistical significance was set at $P<0.05$. The normality of data was verified using the Shapiro-Wilk test. All biomarkers' results, except AOPP, were not normally distributed. Analysis of variance (ANOVA) of repeated measures with Dunn's multiple comparisons test was used to compare the non-parametric results of the sequential time samplings during the study. The ANOVA followed by Tukey's multiple comparison test was applied to study the possible presence of differences between AOPP results. Spearman's correlation coefficient $(r)$ was calculated between the oxidative stress biomarkers with cortisol and the oxytocin values obtained from these animals in a previous report [16] (W0, median: $780 \mathrm{pg} / \mathrm{mL}$; $\mathrm{W}+2$, median: $795 \mathrm{pg} / \mathrm{mL} ; \mathrm{G}+4$, median: $18.6 \mathrm{pg} / \mathrm{mL}$ ). An $\mathrm{r}$ value of $<0.3$ was considered a negligible correlation, following the rule of thumb [51]. Results were described as median and 25th-75th percentile (in the text) and line-box plots (in Figures).

\section{Abbreviations}

ABTS: 2,2'-azino-bis(3-ethylbenz-thiazoline-6-sulfonic acid; ANOVA: Analysis of variance; AOPP: Advanced oxidation protein products; CUPRAC: Cupric reducing antioxidant capacity; FOX: Ferrous oxidation-xylenol orange; FRAS: Ferric reducing ability of saliva; $\mathrm{H}_{2} \mathrm{O}_{2}$ : Hydrogen peroxide; HRP: Horseradish peroxidase; POX-Act: Peroxide-activity; ROS: Reactive oxygen species; TEAC: Trolox equivalent antioxidant capacity; TMB: 3,5,3'5'-tetramethylbenzidine..

\section{Supplementary Information}

The online version contains supplementary material available at https://doi. org/10.1186/s12917-021-03087-2.

Additional file 1. Description of the chemical composition of the dry matter (DM) basis of the starter offered to calves in the experiment.

\section{Acknowledgements}

Not applicable.

\section{Authors' contributions}

XM, EM, CPR and EN designed the experiments and conceptualised the manuscript. CPR and DE performed the methodology and data analysis. JJC and CPR interpreted the data and wrote the manuscript. All authors have read and approved the final manuscript.

\section{Funding}

CPR has a post-doctoral fellowshisp "Juan de la Cierva Formación" supported by the "Ministerio de Economía y Competitividad" (FJC2019-042475-I), Spain.

Availability of data and materials

The datasets used and/or analysed during the current study are available from the corresponding author on reasonable request.

\section{Declarations}

Ethics approval and consent to participate

All experimental procedures described in this study were approved by the Institutional Animal Care and Use Committee of the Autonomous University of Barcelona (Spain) (CEEA-3621) and were in strict accordance with the Ethics Procedures of the European directive 2010/63/EU. The informed consent to participate from farm owners for using animals in the study was obtained in all the trials of the study.

\section{Consent for publication}

Not applicable.

\section{Competing interests}

The authors declare that they have no competing interests.

\section{Author details}

${ }^{1}$ Department of Animal and Food Science, School of Veterinary Science, Universitat Autònoma de Barcelona, 08193 Cerdanyola del Vallès, BarceIona, Spain. ${ }^{2}$ Interdisciplinary Laboratory of Clinical Analysis (Interlab-UMU), Veterinary School, Campus of Excellence Mare Nostrum, University of Murcia, Campus de Espinardo s/n, 30100 Murcia, Spain.

Received: 19 July 2021 Accepted: 18 November 2021

Published online: 04 December 2021

\section{References}

1. Sies H, Jones DP. Reactive oxygen species (ROS) as pleiotropic physiological signalling agents. Nat Rev Mol Cell Biol. 2020;21:363-83. https://doi. org/10.1038/s41580-020-0230-3. 
2. D'Odorico S, Bortolan R, Cardin A. Reduced plasma antioxidant concentrations and increased oxidative DNA damage in inflammatory bowel disease. Scand J Gastroenterol. 2009;36:1289-94. https://doi.org/10.1080/ 003655201317097146.

3. Quintavalla F, Basini G, Bussolati S, Carrozzo GG, Inglese A, Ramoni R. Redox status in canine leishmaniasis. Animals. 2021;11:1-10.

4. Song YR, Kim JK, Lee HS, Kim SG, Choi EK. Serum levels of protein carbonyl, a marker of oxidative stress, are associated with overhydration, sarcopenia and mortality in hemodialysis patients. BMC Nephrol. 2020;21:1-11

5. Song Y, Loor JJ, Li C, Liang Y, Li N, Shu X, et al. Enhanced mitochondrial dysfunction and oxidative stress in the mammary gland of cows with clinical ketosis. J Dairy Sci. 2021;104:6909-18.

6. Heidarpour M, Mohri M, Fallah-Rad AH, Shahreza FD, Mohammadi M. Oxidative stress and trace elements before and after treatment in dairy cows with clinical and subclinical endometritis. Rev Med Vet (Toulouse), 2012;163:628-33.

7. Talukder S, Ingenhoff L, Kerrisk KL, Celi P. Plasma oxidative stress biomarkers and progesterone profiles in a dairy cow diagnosed with an ovarian follicular cyst. Vet Q. 2014;34:113-7. https://doi.org/10.1080/01652176. 2014.953264.

8. Uzlu E, Karapehlivan M, Erdoğan HM, Kiziltepe Ş, Erkiliç EE, Deveci $H A$, et al. Serum and saliva sialic acid and oxidative stress parameters changes in bulls with foot and mouth disease. Kafkas Univ Vet Fak Derg. 2016;22:321-5.

9. Dimitrijević B, Borozan S, Katić-Radivojević S, Stojanović S. Effects of infection intensity with Strongyloides papillosus and albendazole treatment on development of oxidative/nitrosative stress in sheep. Vet Parasitol. 2012;186:364-75.

10. Aktas MS, Kandemir FM, Kirbas A, Hanedan B, Aydin MA. Evaluation of oxidative stress in sheep infected with Psoroptes ovis using total antioxidant capacity, total oxidant status, and malondialdehyde level. J Vet Res. 2017:61:197-201.

11. Sies $\mathrm{H}$. On the history of oxidative stress: concept and some aspects of current development. Curr Opin Toxicol. 2018;7:122-6. https://doi.org/10. 1016/j.cotox.2018.01.002.

12. Li H, Zhang Y, Li R, Wu Y, Zhang D, Xu H, et al. Effect of seasonal thermal stress on oxidative status, immune response and stress hormones of lactating dairy cows. Anim Nutr. 2021;7:216-23. https://doi.org/10.1016/j. aninu.2020.07.006.

13. Putman AK, Brown JL, Gandy JC, Wisnieski L, Sordillo LM. Changes in biomarkers of nutrient metabolism, inflammation, and oxidative stress in dairy cows during the transition into the early dry period. J Dairy Sci. 2018;101:9350-9. https://doi.org/10.3168/jds.2018-14591.

14. Hefnawy A, Helal MAY, Sabek A, Shousha S. Clinical, behavioral and biochemical alterations due to shearing stress in Ossimi sheep. J Vet Med Sci. 2018:80:1281-6. https://doi.org/10.1292/jvms.18-0150.

15. Aschbacher K, O'Donovan A, Wolkowitz OM, Dhabhar FS, Su Y, Epel E. Good stress, bad stress and oxidative stress: insights from anticipatory cortisol reactivity. Psychoneuroendocrinology. 2013;38:1698-708. https:// doi.org/10.1016/j.psyneuen.2013.02.004

16. López-Arjona M, Mainau E, Navarro E, Contreras-Aguilar MD, Escribano D, Mateo SV, et al. Oxytocin in bovine saliva: validation of two assays and changes in parturition and at weaning. BMC Vet Res. 2021;17:1-8.

17. Al-Rawi NH, Shahid AM. Oxidative stress, antioxidants, and lipid profile in the serum and saliva of individuals with coronary heart disease: is there a link with periodontal health? Minerva Stomatol. 2017;66:212-25. https:// doi.org/10.23736/S0026-4970.17.04062-6.

18. Escribano D, Soler L, Gutiérrez AM, Martínez-Subiela S, Cerón JJ. Measurement of chromogranin a in porcine saliva: validation of a time-resolved immunofluorometric assay and evaluation of its application as a marker of acute stress. Animal. 2013;7:640-7.

19. Rubio CP, Contreras-Aguilar MD, Quiles A, López-Arjona M, Cerón JJ, Martínez-Subiela S, et al. Biomarkers of oxidative stress in saliva of sheep: analytical performance and changes after an experimentally induced stress. Res Vet Sci. 2019;123:71-6. https://doi.org/10.1016/j.rvsc.2018.12. 015.

20. Klimiuk A, Zalewska A, Sawicki R, Knapp M, Maciejczyk M. Salivary oxidative stress increases with the progression of chronic heart failure. J Clin Med. 2020;9:769.
21. Puzio W, Chrobak L, Rutkowski M, Franczyk M, Kankofer M. Antioxidative and oxidative profiles in plasma and saliva of cows in different ages and hormonal status. Vet Ital. 2019;55:319-26.

22. Contreras-Aguilar MD, Vallejo-Mateo PJ, Lamy E, Escribano D, Cerón JJ, Tecles F, et al. Changes in saliva analytes in dairy cows during peripartum: a pilot study. Animals. 2021;11:1-10.

23. Bolt SL, Boyland NK, Mlynski DT, James R, Croft DP. Pair housing of dairy calves and age at pairing: effects on weaning stress, health, production and social networks. PLoS One. 2017;12:1-18.

24. Jasper J, Budzynska M, Weary DM. Weaning distress in dairy calves: acute behavioural responses by limit-fed calves. Appl Anim Behav Sci. 2008;110:136-43.

25. Weary DM, Jasper J, Hötzel MJ. Understanding weaning distress. Appl Anim Behav Sci. 2008;110:24-41.

26. Bùe KE, Gry F. Grouping and social preferences in calves, heifers and cows. Appl Anim Behav Sci. 2003;80:175-90.

27. Kondo S, Kawakami N, Kohama H, Nishino S. Changes in activity, spatial pattern behavior in calves after grouping and social. Appl Anim Ethol. 1984;11:217-28.

28. Pagano G, Talamanca AA, Castello G, Cordero MD, D'ischia M, Gadaleta MN, et al. Oxidative stress and mitochondrial dysfunction across broadranging pathologies: toward mitochondria-targeted clinical strategies. Oxid Med Cell Longev. 2014. https://doi.org/10.1155/2014/541230.

29. Kumar A, Kaur G, Rinwa P. Buspirone along with melatonin attenuates oxidative damage and anxiety-like behavior in a mouse model of immobilization stress. Chin J Nat Med. 2014;12:582-9.

30. McIntosh LJ, Sapolsky RM. Glucocorticoids increase the accumulation of reactive oxygen species and enhance adriamycin-induced toxicity in neuronal culture. Exp Neurol. 1996;141:201-6.

31. Wernicki A, Urban-Chmiel R, Kankofer M, Mikucki P, Puchalski A, Tokarzewski S. Evaluation of plasma cortisol and TBARS levels in calves after short-term transportation. Rev Med Vet (Toulouse). 2006;157:30-4.

32. Ishida N, Hobo S, Takahashi T, Nanbo Y, Sato F, Hasegawa T, et al. Chronological changes in superoxide-scavenging ability and lipid peroxide concentration of equine serum due to stress from exercise and transport. Equine Vet J Suppl. 1999;30:430-3.

33. Fontella FU, Siqueira IR, Vasconcellos APS, Tabajara AS, Netto CA, Dalmaz C. Repeated restraint stress induces oxidative damage in rat hippocampus. Neurochem Res. 2005;30:105-11.

34. Oishi K, Yokoi M, Maekawa S, Sodeyama C, Shiraishi T, Kondo R, et al. Oxidative stress and haematological changes in immobilized rats. Acta Physiol Scand. 1999;165:65-9.

35. Ferreira CS, Vasconcellos RS, Pedreira RS, Silva FL, Sá FC, Kroll FSA, et al. Alterations to oxidative stress markers in dogs after a short-term stress during transport. J Nutr Sci. 2014;3:e27. https://doi.org/10.1017/jns.2014. 47.

36. Bernabucci U, Ronchi B, Lacetera N, Nardone A. Markers of oxidative status in plasma and erythrocytes of transition dairy cows during hot season. J Dairy Sci. 2002;85:2173-9. https://doi.org/10.3168/jds.S00220302(02)74296-3.

37. Majlesi A, Yasini SP, Azimpour S, Mottaghian P. Evaluation of oxidative and antioxidant status in dairy calves before and after weaning. Bulg J Vet Med. 2021;24:184-90

38. López-Arjona M, Escribano D, Mateo SV, Contreras-Aguilar MD, Rubio $C P$, Tecles $F$, et al. Changes in oxytocin concentrations in saliva of pigs after a transport and during lairage at slaughterhouse. Res Vet Sci. 2020;133(August):26-30. https://doi.org/10.1016/j.rvsc.2020.08.015.

39. Escribano D, Fuentes-Rubio M, Cerón JJ. Validation of an automated chemiluminescent immunoassay for salivary cortisol measurements in pigs. J Vet Diagnostic Investig. 2012;24:918-23.

40. Escribano D, Gutiérrez AM, Tecles F, Cerón JJ. Changes in saliva biomarkers of stress and immunity in domestic pigs exposed to a psychosocial stressor. Res Vet Sci. 2015;102:38-44. https://doi.org/10.1016/j.rvsc.2015. 07.013 .

41. Contreras-Aguilar MD, Escribano D, Martínez-Subiela S, Martínez-Miró S, Rubio M, Tvarijonaviciute A, et al. Influence of the way of reporting alphaamylase values in saliva in different naturalistic situations : a pilot study. PLoS One. 2017:12:1-13.

42. González-Hernández JM, Franco L, Colomer-Poveda D, Martinez-Subiela S, Cugat R, Cerón JJ, et al. Influence of sampling conditions, salivary flow, 
and total protein content in uric acid measurements in saliva. Antioxidants. 2019;8:2-9.

43. Percie Du Sert N, Hurst V, Ahluwalia A, Alam S, Avey MT, Baker M, et al. The ARRIVE guidelines 2.0: updated guidelines for reporting animal research. BMJ Open Sci. 2020:4:e100115.

44. Council, N. Nutrient requirements of dairy cattle. National Research. 2001;319.

45. Campos C, Guzmán R, López-Fernández E, Casado Á. Evaluation of the copper(II) reduction assay using bathocuproinedisulfonic acid disodium salt for the total antioxidant capacity assessment: the CUPRAC-BCS assay. Anal Biochem. 2009;392:37-44.

46. Benzie IF, Strain JJ. The ferric reducing ability of plasma (FRAP) as a measure of "antioxidant power": the FRAP assay. Anal Biochem. 1996;239:70-6. https://doi.org/10.1006/abio.1996.0292.

47. Arnao MB, Cano A, Hernández-Ruiz J, Garcıa-Cánovas F, Acosta M. Inhibition byl-ascorbic acid and other antioxidants of the 2,2'-Azino-bis(3ethylbenzthiazoline-6-sulfonic acid) oxidation catalyzed by peroxidase: a new approach for determining Total antioxidant status of foods. Anal Biochem. 1996;236:255-61. https://doi.org/10.1006/abio.1996.0164.

48. Witko-Sarsat V, Friedlander M, Capeillère-Blandin C, Nguyen-Khoa T, Nguyen AT, Zingraff J, et al. Advanced oxidation protein products as a novel marker of oxidative stress in uremia. Kidney Int. 1996;49:1304-13. https://doi.org/10.1038/ki.1996.186.

49. Arab K, Steghens JP. Plasma lipid hydroperoxides measurement by an automated xylenol orange method. Anal Biochem. 2004;325:158-63. https://doi.org/10.1016/j.ab.2003.10.022.

50. Tatzber F, Griebenow S, Wonisch W, Winkler R. Dual method for the determination of peroxidase activity and total peroxides-iodide leads to a significant increase of peroxidase activity in human sera. Anal Biochem. 2003;316:147-53. https://doi.org/10.1016/S0003-2697(02)00652-8.

51. Hinkle DE, Wiersma W, Jurs SG. Applied statistics for the behavioral sciences. Boston, Mass: Houghton Mifflin; 2003.

\section{Publisher's Note}

Springer Nature remains neutral with regard to jurisdictional claims in published maps and institutional affiliations.

Ready to submit your research? Choose BMC and benefit from:

- fast, convenient online submission

- thorough peer review by experienced researchers in your field

- rapid publication on acceptance

- support for research data, including large and complex data types

- gold Open Access which fosters wider collaboration and increased citations

- maximum visibility for your research: over $100 \mathrm{M}$ website views per year

At BMC, research is always in progress.

Learn more biomedcentral.com/submissions 